Check for updates

Cite this: RSC Adv., 2017, 7, 51324

Received 28th September 2017 Accepted 26th October 2017

DOI: $10.1039 / \mathrm{c} 7 \mathrm{ra10756k}$

rsc.li/rsc-advances

\section{Characterization of an archaeal photoreceptor/ transducer complex from Natronomonas pharaonis assembled within styrene-maleic acid lipid particles}

N. Voskoboynikova, (D) $\dagger^{a}$ W. Mosslehy, (D) $\dagger^{\mathrm{a}}$ A. Colbasevici, (D) a T. T. Ismagulova, (D) b D. V. Bagrov, (D) ${ }^{\text {b }}$ A. A. Akovantseva, (D) d P. S. Timashev, (DD ${ }^{c d}$ A. Y. Mulkidjanian, (D) a

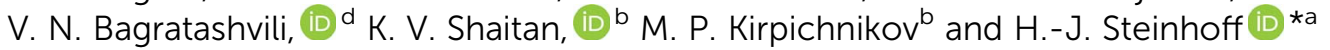

The styrene-maleic acid (SMA) copolymers enable a detergent-free extraction of membrane proteins from lipid bilayers yielding stable water-soluble nanocontainers with lipid-encased proteins. Here, we demonstrate the ability of the SMA copolymer to extract a complex of interacting membrane proteins from proteoliposomes, namely the archaeal sensory rhodopsin II from Natronomonas pharaonis, NpSRII, in complex with its transducer, NpHtrll. The NpSRII/NpHtrll complexes retained their structural integrity within SMA lipid particles as revealed by diverse biophysical techniques. Pulse electron paramagnetic resonance data showed the SMA-encased NpSRII/NpHtrll complexes as native-like 2:2 dimers with a distance between NpSRII sites 159 according to the " $\vee$ "-shaped conformation found in a crystal structure.

\section{Introduction}

The membrane-embedded protein photoreceptor sensory rhodopsin II ( $N p S R I I)$ from the halophilic archaeon Natronomonas pharaonis mediates the photophobic response to harmful blue light. ${ }^{1-3} \mathrm{NpSRII}$ is structurally and functionally related to the light driven ion pumps bacteriorhodopsin (BR), halorhodopsin (HR), and channelrhodopsin. ${ }^{4}$ In addition, NpSRII is distantly related to G-protein coupled receptors (GPCRs) including visual rhodopsins. ${ }^{5}$ Similar to other rhodopsins, NpSRII comprises seven transmembrane helices (A-G) with a retinal chromophore covalently bound to a conserved lysine residue of helix G via a protonated Schiff base. In membranes, $N p S R I I$ interacts with its cognate transducer ( $N p H t r I I)$, which consists of a membrane-embedded $\mathrm{N}$-terminal domain with two transmembrane $\alpha$-helices, TM1 and TM2, and an elongated $\alpha$ helical coiled-coil cytoplasmic signalling domain that protrudes into the cytoplasm by $\sim 26 \mathrm{~nm} .^{6}$ In membrane lipids, the NpSRII/NpHtrII complexes are present in a $2: 2$ molecular stoichiometry. ${ }^{7,8}$ The structure of the transmembrane domains

${ }^{a}$ Department of Physics, University of Osnabrück, 49069, Osnabrück, Germany. E-mail: hsteinho@uos.de

${ }^{b}$ Department of Bioengineering, Faculty of Biology, Lomonosov Moscow State University, 1-12 Leninskiye Gory, 119234, Moscow, Russia

'Institute for Regenerative Medicine of I. M. Sechenov First Moscow State Medical University, 8-2 Trubetskaya St., Moscow, 119991, Russia

${ }^{d}$ Institute of Photonic Technologies of Research Center "Crystallography and Photonics" of RAS, 2 Pionerskaya St., Troitsk, Moscow, 142190, Russia

$\dagger$ N. V. and W. M. contributed equally. of the $N p S R I I / N p H t r I I$ complex, with two receptor molecules flanking a transducer dimer, as initially proposed from electron paramagnetic resonance (EPR) data, ${ }^{9}$ was resolved by X-ray crystallography. ${ }^{1}$

Owing to the availability of the crystal structure, ${ }^{1}$ the possibility to trigger the reaction cycle of NpSRII by a short laser flash, and the homology between $N p H$ trII and other prokaryotic sensory transducers, ${ }^{\mathbf{1 0}}$ the $\mathrm{NpSRII/NpHtrII}$ complex serves as a paradigmal system for studying sensory transduction. ${ }^{\mathbf{1 1}}$ Isomerization of the retinal chromophore in response to light activation of NpSRII leads to an outward movement of helix $\mathrm{F}^{\mathbf{7 , 9 , 1 2 , 1 3}}$ which induces a rotary/screw-like motion of the transducer helix TM2 that may be accompanied by a displacement, ${ }^{\mathbf{9}, 14,15}$ presumably analogous to the piston-like motion in chemoreceptors. ${ }^{\mathbf{1 6 - 1 8}}$ These transient changes in the transmembrane part of $\mathrm{NpHtrII}$ lead to subsequent signal propagating along the cytoplasmic part of $N p H$ trII to the intracellular signalling pathway, ${ }^{19}$ which modulates the cell motility similarly to the bacterial two-component chemotaxis systems (reviewed e.g. in ref. 6, 10 and 20-22). In cell membranes, the $2: 2 \mathrm{NpSRII} / \mathrm{NpHtrII}$ complexes are likely further organized in trimers, ${ }^{19,23,24}$ similarly to the $E$. coli chemoreceptors, ${ }^{25}$ which hinders investigation of protein interactions within separate $N p$ SRII/NpHtrII complexes. Still, the inter-protein interaction within the $2: 2$ photoreceptor/transducer complexes are of considerable interest for elucidation of the signalling pathway.

To facilitate studying of separate 2:2 photoreceptor/ transducer complexes in a lipid environment, we have recently established reconstitution of the detergent-solubilized 
NpSRII/NpHtrII complex into nanodiscs, self-assembling soluble monodisperse lipid discoidal particles of controlled nanometer-scale size, where a fragment of lipid bilayer is surrounded and stabilized by an amphiphilic helical protein belt. ${ }^{26}$ We used single-molecule (SM) fluorescence imaging, fluorescence cross-correlation spectroscopy (FCCS) analysis, and sitedirected spin labeling (SDSL) electron paramagnetic resonance (EPR) spectroscopy to confirm the reconstitution of the 2:2 NpSRII/NpHtrII complex into nanodiscs. Photocycle measurements confirmed functional activity of the reconstituted NpSRII. ${ }^{26}$

Recently, lipid discoidal particles stabilized by amphipathic styrene-maleic acid (SMA) copolymers were suggested as alternative nanocontainers for membrane proteins. While preparation of nanodiscs implies application of detergents, the treatment by SMA copolymers enables a direct, detergent-free extraction of membrane proteins both from artificial and natural lipid bilayers providing stable membrane patches with incorporated protein(s) (reviewed, e.g., in ref. 27-29). SMAencased nanolipoprotein particles are comprised of lipid/ protein cores surrounded by a polymer belt and have, depending on the preparation routine, diameters of 10-30 $\mathrm{nm}$. The SMA copolymer with a $3: 1$ styrene-to-maleic acid molar ratio (3:1 SMA) was shown to extract integral membrane proteins, both $\alpha$-helical bundle proteins such as bacteriorho$\operatorname{dopsin}^{30,31}$ and $\beta$-barrel proteins, such as lipid A palmitoyltransferase PagP $^{30}$ from 1,2-dimyristoyl-sn-glycero-3phosphocholine (DMPC) liposomes. Moreover, the SMA copolymer is able to solubilize, in a detergent-free manner, hetero- and homo-oligomeric membrane protein complexes. ${ }^{27,28,32-34}$ The resulting lipoprotein-SMA particles were 11-12 $\mathrm{nm}$ in diameter and maintained the protein integrity and activity. ${ }^{\mathbf{3 0 , 3 1}}$ The $3: 1$ SMA polymer-mediated lipid solubilisation was also shown to be non-selective in regard to the lipid type. ${ }^{35,36}$ The properties of SMA-solubilized membrane proteins were studied using different biophysical methods. The integral membrane protein KCNE1, e.g., extracted by $3: 1$ SMA from 1-palmitoyl-2-oleoyl-phosphatidylcholine/1-palmitoyl-2oleoyl-phosphatidylglycerol (POPC/POPG) liposomes could be characterized by electron paramagnetic resonance (EPR) spectroscopy with significantly improved resolution when compared to micelles. ${ }^{37,38}$

Here, we have tested whether SMA copolymer can solubilize an archaeal photoreceptor-transducer complex in the absence of detergents. We have shown that the 3:1 SMA copolymer is able to extract, in a detergent-free manner, the $N p S R I I / N p$ HtrII complex from protein-containing liposomes (proteoliposomes) made of $E$. coli polar lipids. The obtained $N p$ SRII/N $p$ HtrII-SMA lipid particles (SMALPs) were characterized by dynamic light scattering (DLS), transmission electron microscopy (TEM), atomic force microscopy (AFM), resonance Raman spectroscopy, as well as by continuous wave (cw) and pulse EPR spectroscopy. NpSRII/NpHtrII was found to be present in SMALPs as structurally integer separate complexes with a photoreceptor: transducer stoichiometry of $2: 2$.

\section{Materials and methods}

The polar lipid extract of $E$. coli membranes in chloroform was purchased from Avanti Polar Lipids, Inc. (Alabaster; AL; USA). $N$ Dodecyl- $\beta$-D-maltoside (DDM) was bought from Anatrace (Affymetrix, Cleveland, Ohio, US). Isopropyl- $\beta$-D-thiogalactopyranosid (IPTG) was acquired from Carl Roth (Karlsruhe, Germany). EDTA-free complete protease inhibitor cocktail was procured from Roche Life Science (Mannheim, Germany). NiNTA superflow agarose was purchased from Qiagen (Hilden, Germany). The styrene maleic acid (SMA) copolymer with styrene-to-maleic acid molar ratio of $3: 1\left(M_{\mathrm{W}} 9500 \mathrm{Da}\right.$, supplied as an aqueous sodium salt solution SMA $3000 \mathrm{HNa}$ ) was kindly provided as a gift by Cray Valley (Exton; PA; USA). All other reagents were of analytical grade.

Protein concentrations were determined using an UV-vis spectrophotometer (UV-2450, Shimadzu Corporation, Kyoto, Japan). The concentration of $N p$ SRII was determined by using the known extinction coefficient of $50000 \mathrm{M}^{-1} \mathrm{~cm}^{-1}$ at $\lambda=$ $280 \mathrm{~nm}$. The concentration of $\mathrm{NpHtrII}_{157}$ was determined according to ref. 39. The concentration of MSP1E3D1 was determined by using the known extinction coefficient of $29400 \mathrm{M}^{-1} \mathrm{~cm}^{-1}$ at $\lambda=280 \mathrm{~nm}$.

\section{Protein expression and purification}

For purification purposes, all proteins had a C-terminal 6xHistag. NpSRII-His and its respective cysteine mutant (NpSRIIL159C) and $N p \mathrm{HtrII}_{157}$-His (transducer construct that was truncated at position 157) were expressed in E. coli BL21 (DE3) cells and purified according to ref. 23, 40 and 41 with minor modifications. Briefly, transformed cells were grown in LuriaBertani (LB) medium containing $50 \mathrm{mg} \mathrm{ml}{ }^{-1}$ kanamycin at $37{ }^{\circ} \mathrm{C}$ to an optical density $\mathrm{OD}_{580}$ of 1.0 . The overexpression of the protein was induced by addition of IPTG to a final concentration of $0.5 \mathrm{mM}$. In the case of $N p S R I I, 10 \mu \mathrm{M}$ all-trans retinal (Sigma) was also added. After an induction period of $3 \mathrm{~h}$ at $37^{\circ} \mathrm{C}$, cells were harvested $\left(4200 \times g ; 15 \mathrm{~min} ; 4^{\circ} \mathrm{C}\right)$, washed and resuspended (1/100 culture volume) in $150 \mathrm{mM} \mathrm{NaCl}$, $25 \mathrm{mM}$ NaPi (pH 8.0), 2 mM EDTA buffer containing a protease inhibitor mix. Cells were disrupted by sonication (Branson Sonifier II W-250, Heinemann, Germany). Membranes were isolated by centrifugation $\left(50000 \times g ; 1 \mathrm{~h} ; 4{ }^{\circ} \mathrm{C}\right)$, and solubilized in buffer A (300 mM NaCl, $50 \mathrm{mM} \mathrm{NaPi}$ (pH 8.0), $2 \%(\mathrm{w} / \mathrm{v}$ ) DDM) overnight at $4{ }^{\circ} \mathrm{C}$. Solubilized membrane proteins were isolated by centrifugation $\left(50000 \times g ; 1 \mathrm{~h} ; 4{ }^{\circ} \mathrm{C}\right)$ followed by chromatography using Ni-NTA superflow material which was pre-equilibrated with buffer B (300 mM NaCl, $50 \mathrm{mM} \mathrm{NaPi} \mathrm{(pH}$ 8.0), $0.05 \%$ (w/v) DDM). Non-specifically bound proteins were removed by washing extensively with buffer B containing $30 \mathrm{mM}$ imidazole. His-tagged proteins were eluted with buffer B containing $200 \mathrm{mM}$ imidazole. Fractions containing the desired protein were pooled and dialyzed against buffer $\mathrm{C}$ (500 mM NaCl, $10 \mathrm{mM}$ Tris (pH 8.0), 0.05\% (w/v) DDM) to remove imidazole. If not used directly for spin labeling and reconstitution, protein samples were flash frozen and stored at $-80{ }^{\circ} \mathrm{C}$. 
MSP1E3D1 was expressed in E. coli BL21 (DE3) cells and was purified according to ref. 42 with minor modifications. Briefly, transformed cells were grown in LB medium containing $50 \mathrm{mg}$ $\mathrm{ml}^{-1}$ kanamycin at $37^{\circ} \mathrm{C}$ to an optical density $\mathrm{OD}_{580}$ of 1.0. The overexpression of the protein was induced by addition of IPTG to a final concentration of $0.5 \mathrm{mM}$. After an induction period of $4 \mathrm{~h}$ at $37^{\circ} \mathrm{C}$, cells were harvested $\left(4200 \times g ; 15 \mathrm{~min} ; 4^{\circ} \mathrm{C}\right)$ and resuspended (1/100 culture volume) in buffer $\mathrm{D}(300 \mathrm{mM} \mathrm{NaCl}$, $40 \mathrm{mM}$ Tris ( $\mathrm{pH}$ 8.0)) containing 1\% Triton. Cells were disrupted by sonication (Branson Sonifier II W-250). The cell suspension was cleared by centrifugation $(50000 \times g ; 45 \mathrm{~min}$; $4{ }^{\circ} \mathrm{C}$ ). The cleared supernatant was subsequently loaded on NiNTA superflow material which was pre-equilibrated with buffer D. The column was washed with 4 bed volumes of each of the following: (1) buffer D containing 1\% Triton; (2) buffer D containing $50 \mathrm{mM}$ cholate; (3) buffer D; (4) buffer D containing $50 \mathrm{mM}$ imidazole. MSP1E3D1 was eluted with buffer D containing $300 \mathrm{mM}$ imidazole. Fractions containing MSP1E3D1 were pooled and dialyzed against $100 \mathrm{mM} \mathrm{NaCl}, 10 \mathrm{mM}$ Tris (pH 8.0) buffer to remove imidazole. If not used directly for reconstitution, protein samples were flash frozen and stored at $-80{ }^{\circ} \mathrm{C}$.

\section{Preparation of proteoliposomes}

E. coli polar lipids were transferred to a glass flask, and chloroform was removed by drying under nitrogen flow. After evaporation of chloroform, the lipid film was dried under vacuum for at least 1 hour, and then hydrated in buffer $\mathrm{E}$ $(150 \mathrm{mM} \mathrm{NaCl}, 10 \mathrm{mM}$ Tris (pH 8.0)) followed by $5 \times$ freeze-thaw cycles and, if not used directly, stored as aliquots at $-80{ }^{\circ} \mathrm{C}$. Before reconstitution, the suspension of liposomes was passed at least $11 \times$ through polycarbonate unipore membranes (Whatman) with a pore size of $400 \mathrm{~nm}$ or $200 \mathrm{~nm}$ using a MiniExtruder Set (Avanti Polar Lipids, Alabaster, AL, USA).

For reconstitution of the $N p S R I / N p H_{t r I I} I_{157}$ complex in liposomes, $N p$ SRII and $N p \mathrm{HtrII}_{157}$ were mixed at a $1: 1$ molar ratio and then incubated for $20 \mathrm{~min}$ on ice followed by mixing with liposomes at a protein-to-lipid molar ratio of $1: 172$. The assembly mixture was subsequently incubated with Bio-Beads SM-2 (Bio-Rad Laboratories; Munich; Germany) (50 mg of beads/mg of detergent) pre-washed extensively with methanol and water. Finally, proteoliposomes were pelleted by centrifugation $\left(15800 \times g ; 15 \mathrm{~min} ; 4^{\circ} \mathrm{C}\right)$ and resuspended in buffer $\mathrm{E}$ at a final lipid concentration of $20 \mathrm{mg} \mathrm{ml}^{-1}$.

\section{Preparation of SMA lipid particles}

SMA/lipid particles (SMALPs) containing the $N p S R I I / N p H_{t r I I} I_{157}$ complex (in the following abbreviated as $N p \mathrm{SRII} / N p \mathrm{HtrII}_{157^{-}}$ SMALPs) were assembled in vitro according to a protocol adapted from ref. 31 . In the case of extrusion through the membrane with $400 \mathrm{~nm}$ pore size, the suspension of proteoliposomes was sonicated additionally for $30 \mathrm{~min}$ in a bath sonicator at room temperature. $5 \%(\mathrm{w} / \mathrm{v})$ solution of SMA copolymer in buffer $\mathrm{E}$ was added dropwise over 2-3 $\mathrm{min}$ to the proteoliposome suspension to get a final lipid-to-SMA weight ratio of $1: 2.5$. The assembly mixture was allowed to equilibrate for 1 hour at room temperature and then for $16 \mathrm{~h}$ at $4{ }^{\circ} \mathrm{C}$. The resulting samples were centrifuged $\left(126000 \times g ; 30 \mathrm{~min} ; 4^{\circ} \mathrm{C}\right)$ to remove non-solubilized material. Subsequently, SMALP preparations were fractionated on a Superdex 200 10/200 GL column (GE Healthcare) using an ÄKTA Purifier FPLC system (GE Healthcare) with a flow rate of $0.5 \mathrm{ml} \mathrm{min}^{-1}$ at $4{ }^{\circ} \mathrm{C}$.

\section{Preparation of nanodiscs}

Nanodiscs containing the $N p S R I I / N p H_{t r I I} I_{157}$ complex were assembled in vitro according to ref. 26 using MSP1D1E3 as a scaffold protein. The assembly was performed in buffer $\mathrm{E}$ containing $5 \mathrm{mM}$ DDM. $N p$ SRII and $N p \mathrm{HtrII}_{157}$ were mixed at a 1 : 1 molar ratio and then incubated for $20 \mathrm{~min}$ on ice. Polar lipids from $E$. coli membranes and MSP1D1E3 were then added to the $N p S R I / / N p H_{t r I I}{ }_{157}$ complex to give final concentrations of $0.76 \mathrm{mM}$ lipids, $14 \mu \mathrm{M}$ MSP1D1E3 and $7 \mu \mathrm{M} N p S R I I / N p H_{t r I I}{ }_{157}$. After incubation for $1 \mathrm{~h}$ at room temperature, the assembly mixture was incubated with Bio-Beads SM-2 overnight at $4{ }^{\circ} \mathrm{C}$. Non-solubilized proteins and any aggregated material were removed by centrifugation at $126000 \times g$ for $15 \mathrm{~min}$ at $4{ }^{\circ} \mathrm{C}$. Samples were stored at $4{ }^{\circ} \mathrm{C}$ until further use. Subsequently, preparations of nanodiscs were fractionated on a Superdex 200 10/200 GL column using an ÄKTA Purifier FPLC system, with a flow rate of $0.5 \mathrm{ml} \mathrm{min}{ }^{-1}$ at $4{ }^{\circ} \mathrm{C}$.

\section{Site directed spin labeling}

The spin label (1-oxyl-2,2,5,5-tetramethylpyrroline-3-methyl) methanethiosulfonate (MTSSL; Enzo life sciences, NY) was covalently bound to the cysteine residue of NpSRII-L159C as described previously. ${ }^{26}$ Excess label was extensively washed out by using a $10 \mathrm{~K}$ Amicon Ultracel centrifugal filter device (Millipore, Schwalbach, Germany). In the following, the spin label side chain is abbreviated as R1.

\section{Dynamic light scattering}

Dynamic light scattering (DLS) measurements were performed on a Zetasizer Nano ZS (Malvern Instruments, Worcestershire, UK) at $550 \mathrm{~nm}$ and $25{ }^{\circ} \mathrm{C}$. Data represent the average of three sets of 14 runs of $10 \mathrm{~s}$ each. The particle size distribution was obtained by using the ZETASIZER software package ver. 7.02 under the assumption that SMALPs were spherically shaped.

\section{Transmission electron microscopy}

The transmission electron microscopy (TEM) images were obtained on a JEM-2100 (Jeol, Japan) transmission electron microscope operated at $200 \mathrm{kV}$. For TEM imaging, a solution of the SMALPs in $10 \mathrm{mM}$ Tris (pH 8.0), $150 \mathrm{mM} \mathrm{NaCl}$ was deposited on Ted Pella grids treated with a glow discharge device Emitech K100X. A drop of solution was incubated for 1-2 minutes, then treated with $1 \%$ uranyl acetate for 1 minute and dried. The size of SMALPs was estimated using the ImageJ software. $^{43}$ 


\section{Atomic force microscopy}

The atomic force microscopy (AFM) images were obtained on a Solver PRO microscope (NT-MDT, Russian Federation) operating in semicontact mode in buffer and using MSCT-AUHW cantilevers. For AFM imaging, a solution of SMALPs was diluted to final NpSRII concentration of $15 \mu \mathrm{g} \mathrm{ml}{ }^{-1}$ by $10 \mathrm{mM}$ Tris ( $\mathrm{pH}$ 8.0), $150 \mathrm{mM} \mathrm{NaCl}, 10 \mathrm{mM} \mathrm{MgCl}_{2}$ and deposited on freshly cleaved mica. The SMALP sample was incubated for 5 minutes, then mica was washed with $1 \mathrm{ml}$ of the buffer and transferred to the AFM liquid cell and scanned. The obtained AFM images were processed with the Femtoscan Online software.

\section{Resonance Raman spectroscopy}

Resonance Raman (RR) spectra were measured on a Nicolet Almega XR dispersive Raman spectrometer (Thermo Fisher Scientific, USA). Scattering was induced by excitation from the $532 \mathrm{~nm}$ second harmonic of a Nd-YAG laser. The laser beam was directed by a microscope (Olympus BX-51) objective $(100 \times)$ to the sample. The diameter of the focused laser spot on the sample was about $0.6 \mu \mathrm{m}$. The laser power at the sample did not exceed $15 \mathrm{~mW}$. Spectra were recorded in the range of 500$2000 \mathrm{~cm}^{-1}$ with a spectral resolution of $2 \mathrm{~cm}^{-1}$. Each spectrum was obtained by averaging 20 exposures for 2 seconds each. Samples were prepared by drying approximately $2 \mu \mathrm{l}$ of the reconstituted $N p S R I / N p H_{t r I I}{ }_{157}$ complex $\left(0.8 \mathrm{mg} \mathrm{ml}^{-1} N p S R I\right)$ in $10 \mathrm{mM}$ Tris (pH 8.0), $150 \mathrm{mM} \mathrm{NaCl}$ on a SERS (Surface Enhanced Raman Scattering) substrate consisting of carbon nanowalls (CNWs) covered with a layer of gold. CNWs were prepared by plasma-enhanced chemical vapour deposition (PECVD) synthesis according to ref. 44.

\section{Electron paramagnetic resonance spectroscopy}

Continuous wave $(\mathrm{cw})$ electron paramagnetic resonance (EPR) measurements were performed as described previously. ${ }^{45}$ Briefly, cw EPR spectra were recorded at room temperature with a home-made EPR spectrometer equipped with a Bruker dielectric resonator (Bruker Biospin; Germany). The microwave power was set to $1.0 \mathrm{~mW}$, the B-field modulation amplitude was adjusted to $0.15 \mathrm{mT}$, and the sample temperature was stabilized by a gas stream through the resonator to $297 \pm 1 \mathrm{~K}$. Samples were loaded into EPR glass capillaries (0.9 $\mathrm{mm}$ inner diameter) at final protein concentrations of $15-20 \mu \mathrm{M}$ (sample volume of $15 \mu \mathrm{l})$.

For pulsed EPR experiments, samples were concentrated to $\sim 100 \mu \mathrm{M}$, loaded into EPR quartz capillaries $(3 \mathrm{~mm}$ outer diameter; sample volume of $40 \mu \mathrm{l}$ ) and frozen with $20 \%$ glycerol. The spin labeling efficiency determined by cw EPR and absorption spectroscopy was about 75\%. Double electronelectron resonance (DEER) experiments were accomplished at X-band frequencies (9.3-9.4 GHz) with a Bruker Elexsys 580 spectrometer equipped with a Bruker Flexline split-ring resonator ER 4118X-MS3 and a continuous flow helium cryostat (CF935; Oxford Instruments) controlled by an Oxford Intelligent
Temperature Controller ITC 503S. Measurements were performed using the four-pulse DEER sequence. ${ }^{46,47}$

$$
\begin{aligned}
\pi / 2\left(\nu_{\text {obs }}\right)-\tau_{1}-\pi\left(\nu_{\text {obs }}\right) & -\tau^{\prime}-\pi\left(\nu_{\text {pump }}\right)-\left(\tau_{1}+\tau_{2}-\tau^{\prime}\right) \\
& -\pi\left(\nu_{\text {obs }}\right)-\tau_{2}-\text { echo. }
\end{aligned}
$$

A two-step phase cycling $(+\langle x\rangle,-\langle x\rangle)$ was performed on $\pi /$ $2\left(\nu_{\text {obs }}\right)$. Time $\tau^{\prime}$ is varied, whereas $\tau_{1}$ and $\tau_{2}$ are kept constant. The dipolar evolution time is given by $t=\tau^{\prime}-\tau_{1}$. Data were analysed only for $t>0$. The resonator was overcoupled and the pump frequency $\nu_{\text {pump }}$ was set to the center of the resonator dip and coincided with the maximum of the nitroxide EPR spectrum, whereas the observer frequency $\nu_{\text {obs }}$ was $\sim 67 \mathrm{MHz}$ higher (coinciding with the low field local maximum of the spectrum). All measurements were performed at a temperature of $50 \mathrm{~K}$ with observer pulse lengths of $16 \mathrm{~ns}$ for $\pi / 2$ and $32 \mathrm{~ns}$ for $\pi$ pulses and a pump pulse length of 12 ns. Proton modulation was averaged by adding traces at eight different $\tau_{1}$ values, starting at $\tau_{1,0}=200 \mathrm{~ns}$ and incrementing by $\Delta \tau_{1}=8 \mathrm{~ns}$. Data points were collected in $8 \mathrm{~ns}$ time steps. The total measurement time for each sample was $\sim 24 \mathrm{~h}$. Analysis of the data was performed with DeerAnalysis $2013 .^{38,48}$

\section{Rotamer analysis}

Spin label conformations were calculated utilizing the software package MtsslWizard version $1.3,{ }^{49}$ which is a plugin for the PyMOL molecular graphics system. The "tether-in-a-cone" approach $^{50-52}$ was used which means that the program scans for all possible spin label rotamer conformations that do not clash with the given protein structure or with a label itself within a variable tolerance. The following settings were used: label MTSSL; speed - painstaking search; vdW restraints - loose. In the case of the crystal structure published by V. Gordeliy et al. ${ }^{1}$ rotamer analysis was performed based on the relaxed structure $^{19}$ of a dimer of dimers with the transducer truncated at position 157. In process of equilibration, the structure becomes unsymmetrical particularly due to asymmetry of the HAMPdomain (residues $\sim 131-157$ ), which leads to differences in the sets of rotamers from each NpSRII molecule in the complex.

\section{Results}

We utilized the SMA copolymer to isolate a heterogeneous complex of functionally and structurally interacting membrane proteins, NpSRII/NpHtrII, from proteoliposomes without the use of detergent. The resulting nanosized lipoproteincontaining SMA particles were subsequently characterized by a set of biophysical methods.

We first reconstituted the purified detergent-solubilized $N p S R I I / N p H_{t r I I}{ }_{157}$ complex in preformed liposomes of E. coli membrane polar lipids. Addition of the $3: 1$ SMA polymer at a lipid-to-SMA weight ratio of $1: 2.5$ to the suspension of the proteoliposomes obtained was followed by incubation for 1 hour at room temperature and then for $16 \mathrm{~h}$ at $4{ }^{\circ} \mathrm{C}$. The concentration of $2.5 \%(\mathrm{w} / \mathrm{v})$ SMA used was previously reported to be efficient in solubilization of DMPC liposomes. ${ }^{53}$ The 
resulting SMALP preparations were fractionated by sizeexclusion chromatography (SEC). An exemplary SEC purification profile of assembled SMALP particles (Fig. 1A) shows a major peak with the presence of a minor peak in the void volume corresponding to residual aggregated species. The major peak has an absorption maximum at $\sim 500 \mathrm{~nm}$, characteristic for the NpSRII bound retinal chromophore, which coincides with the absorption maximum of the detergentsolubilized NpSRII (Fig. 1B). Fractions from the major peak were pooled and further analysed by dynamic light scattering (DLS) at $550 \mathrm{~nm}$ and $25{ }^{\circ} \mathrm{C}$ to assess the average size of assembled SMALP particles (Fig. 1C) (see Materials and methods). The intensity-weighted particle diameter was estimated to be in the $10 \mathrm{~nm}$ range consistent with previously reported data. ${ }^{28,30,31,54}$ The DLS data indicate the monodisperse character of the SMALP preparations. Thus, incubation of $N p S R I I / N p H_{t r I I}{ }_{157}$-liposomes with $3: 1$ SMA polymer resulted in formation of protein-containing SMA particles.

\section{Transmission electron microscopy imaging}

Visualization of individual SMALPs deposited on the solid support was performed by transmission electron microscopy (TEM) imaging of negatively stained SMALPs. TEM images of SMALP preparations revealed monodisperse nanosized particles with well-defined edges (Fig. 2A). Measurements of 200 individual SMALPs (each particle measured twice) shows that their majority is $\mathbf{1 2 - 1 4} \mathrm{nm}$ in diameter (Fig. 2B) in agreement with results obtained by other authors. ${ }^{31,55}$ These dimensions are larger than those detected by DLS (Fig. 1C) which may be due to the negative staining for TEM imaging. ${ }^{30,56}$ In addition, different physical characteristics were measured by DLS and

(A)

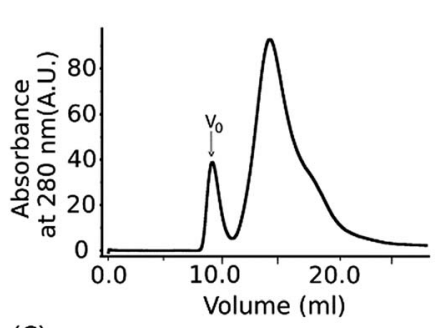

(C)

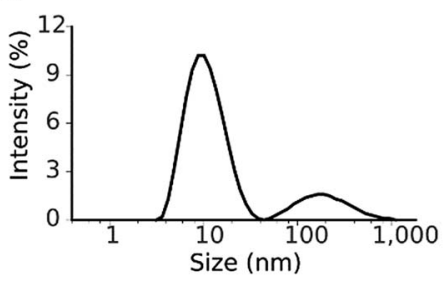

Fig. 1 Physical characterization of the NpSRII/NpHtrll 157 complex upon solubilization with the 3 : 1 SMA copolymer. (A) Size-exclusion chromatography purification profile of SMALP preparations. $V_{0}$ indicates the void volume. (B) Absorption spectrum for the major SEC peak (solid line) and the detergent-solubilized NpSRII/NpHtrll ${ }_{157}$ complex (dashed line), respectively. (C) Size distribution based on DLS data obtained for the major SEC peak.
(A)
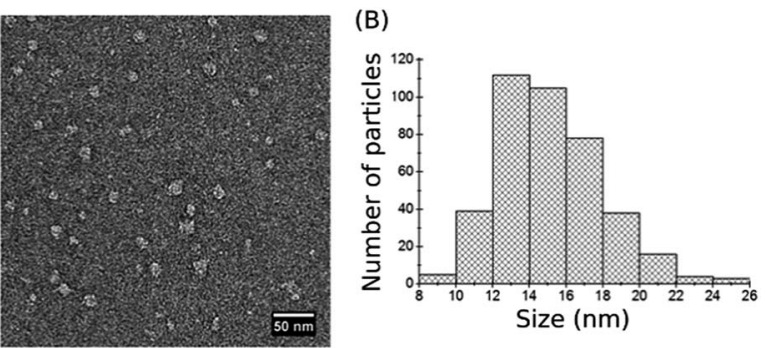

Fig. 2 TEM imaging. (A) Negative-stain TEM image of assembled SMALP particles. (B) Size distribution based on TEM data.

TEM, namely the intensity-weighted diameter in solution versus the size of the projected area of the surface-adsorbed SMALPs.

\section{Atomic force microscopy imaging}

We used atomic force microscopy (AFM) to estimate directly the thickness of assembled SMALPs under conditions similar to those that were shown to be suitable for nanodiscs. ${ }^{42,57,58}$ The AFM images of SMALPs were obtained in semicontact mode; the results are shown in Fig. 3. The mean height of SMALPs is $4.0 \mathrm{~nm}$ which is slightly less than the expected lipid bilayer thickness of about 4.0-5.0 $\mathrm{nm}$. Seemingly, SMALPs either were flattened at the surface and/or compressed by the tip-sample interaction force. Both effects would decrease the measured height. The lateral radius of the SMALPs measured by AFM exceeded $23 \mathrm{~nm}$, which is due to the well-known tip broadening effect of the silicon nitride cantilevers used with a curvature radius of up to $20 \mathrm{~nm}$. The tip broadening effect could be decreased in future measurements by using tips with 1-3 nm radius.

\section{Resonance Raman spectroscopy}

Resonance Raman (RR) spectroscopy is a suitable technique to study the configuration of the retinal chromophore in the
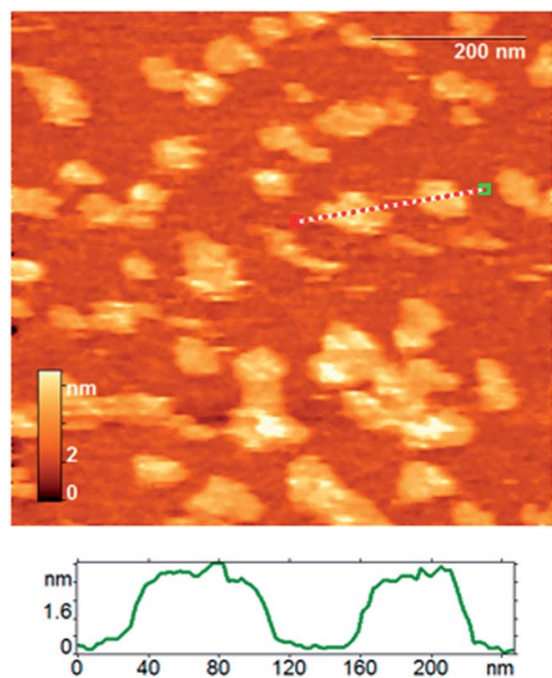

Fig. 3 The AFM height image of assembled SMALP particles; an exemplary section profile is shown below. 


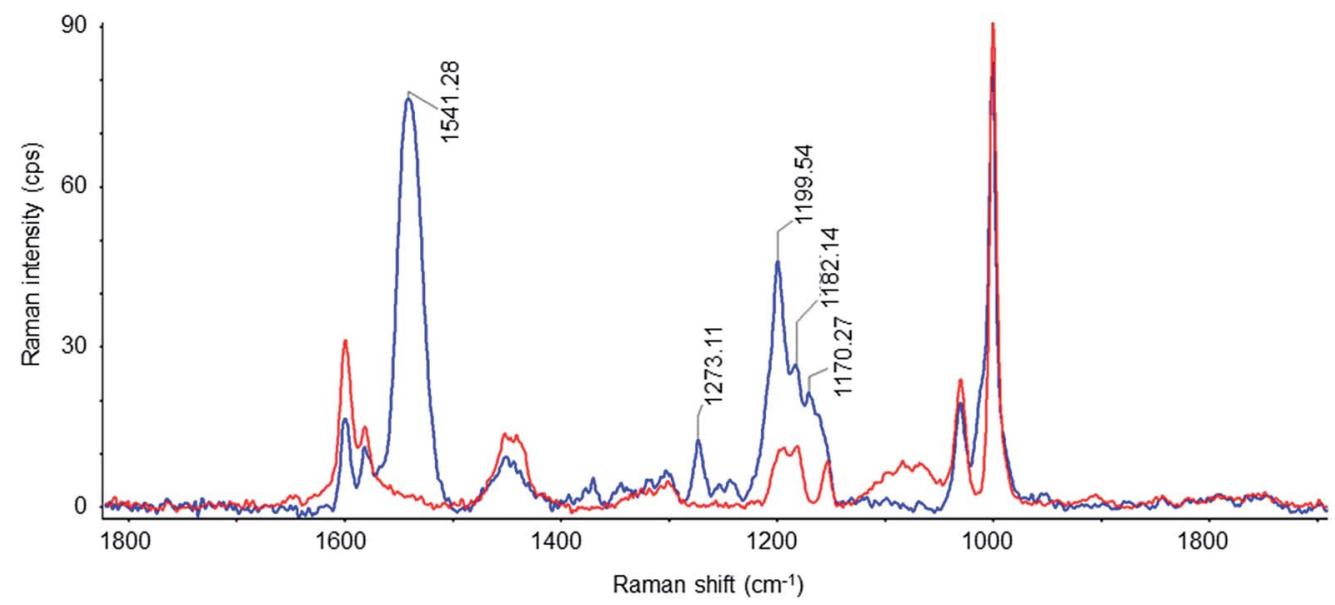

Fig. 4 Resonance Raman spectra from empty SMALPs (red) and SMALPs loaded with the NpSRII/NpHtrll 157 complex (blue) measured with $532 \mathrm{~nm}$ excitation.

protein. RR scattering enables to selectively probe the retinal vibrations because the retinal bands are selectively enhanced due to the resonance effect while the vibrational contributions of the apoprotein are negligible. Fig. 4 shows the RR spectrum of $N p$ SRII/NpHtrII ${ }_{157}$-SMALPs (blue line). To define the possible contributions of empty SMALPs, their RR spectrum (red line) recorded under identical conditions is also shown, being very similar to the known spectra of SMA films. ${ }^{59}$ Vibrational bands of the RR spectrum of empty SMALPs clearly contribute to the RR spectrum of $N p S R I I / N p H_{t r I I}{ }_{157}$-SMALPs, but still enable analysis of crucial regions of the retinal spectrum. The most prominent peak at $1541 \mathrm{~cm}^{-1}$ corresponds to the ethylenic $(\mathrm{C}=\mathrm{C})$ stretching vibration, $\nu_{\mathrm{C}=\mathrm{C}}$, of retinal. The high frequency of this band correlates with the absorption maximum, $\lambda_{\max }$, of $497 \mathrm{~nm}$ in the visible range based on the well-established correlation of $\nu_{\mathrm{C}=\mathrm{C}}$ and $\lambda_{\text {max }}{ }^{\mathbf{6 0}}$ The fingerprint region between 1100 and $1300 \mathrm{~cm}^{-1}$ with the predominant absorption of the $\mathrm{C}-\mathrm{C}$ single bond stretches is known to display the conformation of the retinal polyene chain depending on the isomeric state of the retinal. The corresponding band pattern of NpSRII/

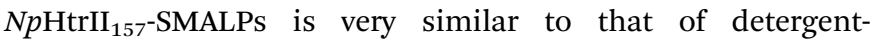
solubilized $N p S R I I^{61}$ and lipid-reconstituted SRII from the halophilic archaeon Halobacterium salinarum ${ }^{62}$ (see Table 1). The characteristic peaks at $1273 \mathrm{~cm}^{-1}, 1200 \mathrm{~cm}^{-1}$ and $1170 \mathrm{~cm}^{-1}$ are similar to those of parent states of bacteriorhodopsin and halorhodopsin, which include an all-trans retinal protonated Schiff base ${ }^{63}$ (see Table 1). The pronounced band at $1200 \mathrm{~cm}^{-1}$ characteristic for C14-C15 stretch vibrations and the band at $1170 \mathrm{~cm}^{-1}$ originating mainly from the $\mathrm{C} 10-\mathrm{C} 11$ stretch vibrations ${ }^{64}$ confirm the predominance of the all-trans retinal conformation. The occurrence of the weak peak at $1182 \mathrm{~cm}^{-1}$ indicates the presence of low amounts of 13-cis retinal. ${ }^{65}$ Thus, the present RR data confirm that reconstituted $N p S R I I$ retains its light-sensitive retinal chromophore, which functional configuration is not affected upon the SMA-mediated extraction of the $\mathrm{NpSRII} / \mathrm{NpH} \mathrm{HtrII}_{157}$ complex.

\section{Electron paramagnetic resonance spectroscopy}

Electron paramagnetic resonance (EPR) spectroscopy is a powerful method to study structural details and conformational transitions of membrane proteins in lipid environments. ${ }^{66,67}$ Continuous wave (cw) EPR spectroscopy allows to probe the spin label side chain dynamics and can provide detailed information on the conformation and conformational dynamics of spin labeled membrane proteins embedded in lipid bilayers. ${ }^{13}$ Pulse double electron-electron resonance (DEER) enables measuring interspin distances in the range of 1.5-8 $\mathrm{nm}$ in site-directed spin labeled proteins, that provide valuable information to characterize the protein structure and structural changes. ${ }^{68,69}$

Table 1 Measured resonance frequencies of vibrational modes and peak assignments for NpSRII compared to some archaeal rhodopsins

\begin{tabular}{|c|c|c|c|c|c|}
\hline Rhodopsin & $\mathrm{C}=\mathrm{C}$ stretch, $\mathrm{cm}^{-1}$ & $\mathrm{C}_{14}-\mathrm{C}_{15}$ stretch, $\mathrm{cm}^{-1}$ & $\mathrm{C}_{10}-\mathrm{C}_{11}$ stretch, $\mathrm{cm}^{-1}$ & $\mathrm{CH}$ rocking, $\mathrm{cm}^{-1}$ & In-plane $\mathrm{C}-\mathrm{CH}_{3}$ rocking, $\mathrm{cm}^{-1}$ \\
\hline$N p$ SRII $^{a}$ (here) & 1541 & 1200 & 1170 & 1273 & $\sim 1000$ \\
\hline SRII ${ }^{b, 61}$ & 1548 & 1202 & & 1274 & 1012 \\
\hline $\mathrm{BR}_{570}^{d, 63}$ & 1529 & 1200 & 1169 & 1273 & 1008 \\
\hline $\mathrm{HR}_{578}{ }^{e, 63}$ & 1522 & 1200 & 1170 & 1272 & 1005 \\
\hline
\end{tabular}

${ }^{a}$ In complex with $\mathrm{NpHtrII}$ in SMALPs. ${ }^{b}$ From Natronomonas pharaonis, detergent-solubilized. ${ }^{c}$ From Halobacterium salinarum (Hs), lipidreconstituted. ${ }^{d}$ From $\mathrm{Hs}$, lipid-reconstituted. ${ }^{e}$ From $\mathrm{Hs}$, detergent-solubilized. 


\section{Continuous wave electron paramagnetic resonance spectroscopy}

The mutant of NpSRII, NpSRII-L159R1 (Fig. 5A), carrying the commonly used nitroxide spin label (1-oxyl-2,2,5,5tetramethylpyrroline-3-methyl)methanethiosulfonate (MTSSL, side chain R1) at position 159, was chosen for EPR measurements. The spin label side chain of NpSRII-L159R1 resides at the cytoplasmic end of helix $\mathrm{F}$ outside of the membrane. $N p$ SRII-L159R1 has been shown to provide a sensitive probe for EPR analyses of conformational changes involved in the light induced signal transfer from NpSRII to NpHtrII. ${ }^{13}$ Due to this property, NpSRII-L159R1 is utilized here to trace the possible impact of the nanoparticle environment on the NpSRII/NpHtrII complex conformation and dynamics. To monitor the spin-label dynamics by room temperature (RT) cw EPR measurements, $N p$ SRII-L159R1 was reconstituted alone or in complex with its cognate transducer $\mathrm{NpHtrII} \mathrm{H}_{157}$, in SMALPs and, for comparison, in liposomes and nanodiscs. The spectra of the L159R1 side chains in the different lipid environments (Fig. 5B) are almost identical revealing a powder-like spectral pattern with a resolved hyperfine line in the high field region of the spectra. Moreover, these spectra are very similar to that of the NpSRIIL159R1/NpHtrII 157 complex reconstituted in native purple membrane lipids (PML). ${ }^{\mathbf{1 3}}$ The presence of the transducer $N p \mathrm{HtrII}_{157}$ does not influence the behaviour of L159R1 (not shown). The spectral shape reveals restriction of the side chain residual motion due to strong sterical interaction between the nitroxide and neighbouring residues of the protein in agreement with the inward facing orientation of the spin label

(A)

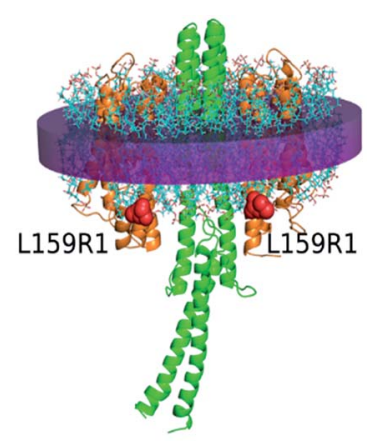

(B)

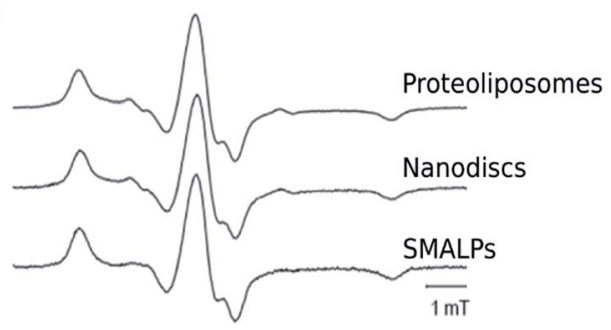

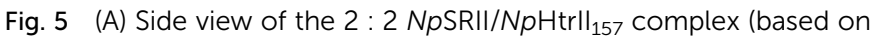
the structural mode ${ }^{19}$ where the intermembrane part corresponds to the crystal structure (PDB: $\left.1 \mathrm{H} 2 \mathrm{~S})^{1}\right)$. The spin labeled residue $\mathrm{L} 159 \mathrm{C}$ is depicted in red. (B) CW EPR spectra revealing the spin label mobility for NpSRII-L159R1/NpHtrll ${ }_{157}$ after reconstitution in proteoliposomes, nanodiscs, and SMALPs. All spectra are normalized to maximum amplitude. binding site (see Fig. 5A and B). Hence, the nitroxide dynamics is not affected significantly by changes of the lipid environment.

\section{Pulse electron paramagnetic resonance spectroscopy}

Four-pulse double electron-electron resonance (DEER) measurements were performed on NpSRII-L159R1 in complex with $N p \mathrm{HtrII}_{157}$ to determine the distribution of distances between the nitroxides in the complex. The DEER data for the complex reconstituted in SMALPs (depicted in blue) or nanodiscs (depicted in red) are shown in Fig. 6. For both SMALP and nanodisc samples, the interspin distance distribution range accessible in the present experiment is between $\sim 1.5$ and $5 \mathrm{~nm}$. The DEER interspin distance distributions for NpSRII-L159R1/ $N p \mathrm{HtrII}_{157}$ reconstituted in SMALPs are characterized by three maxima located at $\sim 2.3,3.7$, and $4.8 \mathrm{~nm}$. These results are similar to the DEER data for the complex in nanodiscs, with maxima at $\sim 2.1,3.6$, and $4.8 \mathrm{~nm}$, indicating occupation of multiple spin label side chain orientations in both cases (Fig. 6E). In nanodiscs these maxima are superimposed to a broad population density ranging from $3.6 \mathrm{~nm}$ down to below $2 \mathrm{~nm}$. Since the minimal interspin distance in the NpSRIIL159R1/ $N p \mathrm{HtrII}_{157}$ complex cannot fall below $2 \mathrm{~nm}$ due to sterical reasons (see below), we have to conclude that these contributions originate from a fraction of nanodiscs containing only NpSRII-L159R1 dimers.

\section{Rotamer analysis}

To compare experimentally determined interspin distances with currently available quaternary structures, ${ }^{\mathbf{1 , 7 0}}$ we performed in silico spin-labeling of two available crystal structures of $N p S R I I$ in complex with the membrane domain of $N p H t r I I$ followed by simulation of the expected interspin distances for the single 2: 2 NpSRII-L159R1/NpHtrII dimer (see Materials and methods). Crystal structures of $N p S R I I$ in complex with truncated transducers in the ground state have been determined in the space groups $P 2_{1} 2_{1} 2$ and $I 2_{1} 2_{1} 2_{1} \cdot{ }^{1,70}$ In both cases X-ray diffraction data showed electron density only for the transmembrane domain of the transducer but not for its adjacent HAMP domain. Although the structures of the rhodopsin and transducer monomers taken individually are nearly the same, the relative orientations of symmetrical parts of the two NpSRII proteins have been found to be parallel, "U"-shaped, in the space group $I 2{ }_{1} 2_{1} 2_{1}$ (PDB: 5JJE), and gusset-like, "V"-shaped, in the space group $P 2{ }_{1} 2_{1} 2$ (PDB: $1 \mathrm{H} 2 \mathrm{~S}$ ). It has been suggested that the transition between "V"- and "U"-shaped conformations could be involved in signal transduction from the receptor to the signalling domain of $\mathrm{NpHtrII} .^{70}$ Here, we simulated interspin distances between spin labeled sites L159R1 for models based on both of these crystal structures. For the "V"-shaped conformation the transducer was complemented by homology modeling of the first HAMP domain and its attachment to the resolved membrane domain of $\mathrm{NpHtrII.{ } ^ { 1 9 }}$ For the "U"-shaped conformation such data were not available so that the interspin calculations were performed in the absence of the HAMP domain. The results are shown in Fig. 6A, B and F, in comparison to the experimental interspin distance distributions 
(A)
(B)

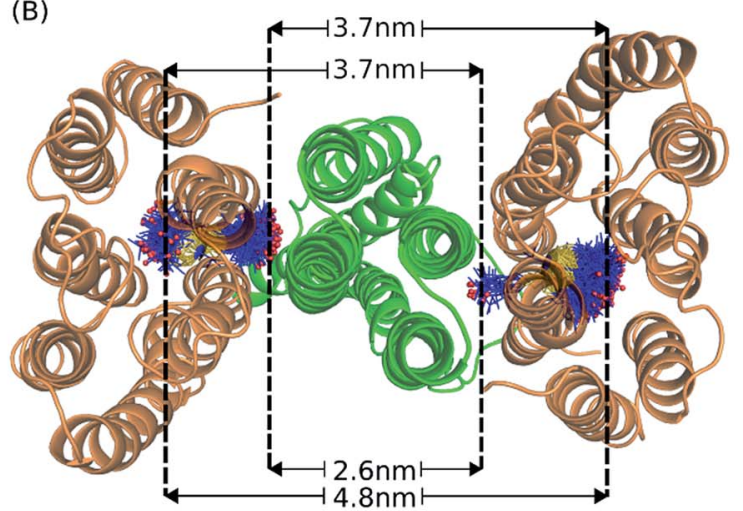

(E)

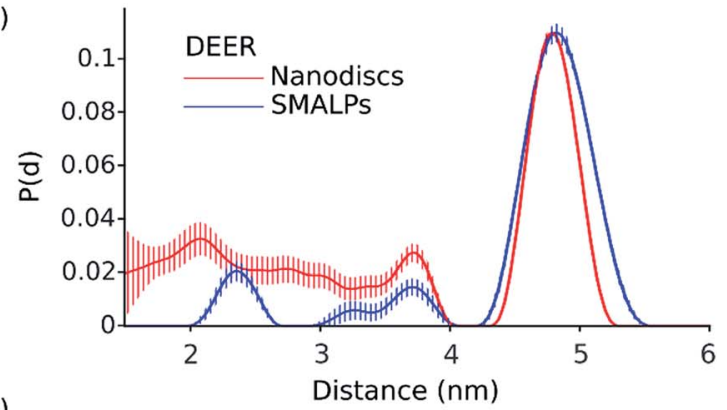

(F)

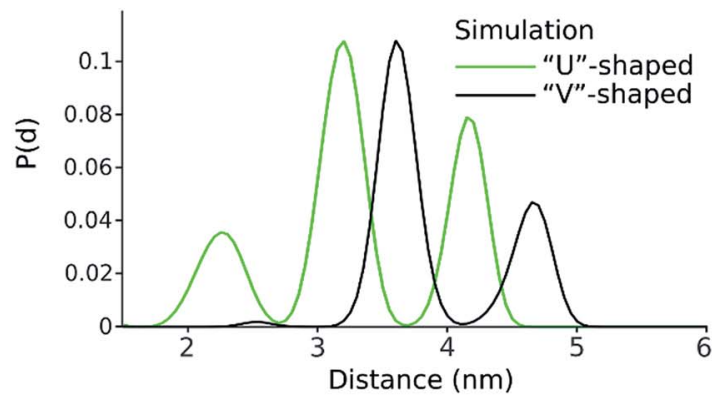

Fig. 6 Structural model, DEER data and distance distribution for the NpSRII-L159R1/NpHtrll 157 complex. (A) Equilibrated model of the NpSRIIL159R1/NpHtrll ${ }_{157}$ complex in "V"-shape conformation based on the crystal structure PDB: 1 H2S. ${ }^{1,19}$ Side view of the protein complex with the membrane borders shown as magenta (extracellular side) and cyan (cytoplasmic side) dotted plates. Spin label conformations resulting from MtssIWizard simulation at the position L159 of NpSRII (orange) are shown as blue sticks with red spheres as nitroxide spin centres. Spin centres are shown more detailed in the zoomed part of the figure. (B) View from the cytoplasm of the model described in (A), where a set of three distance

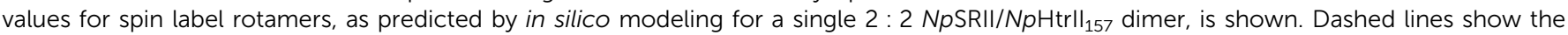
approximate position of the maximum density of the spin centres and help to visualize their possible occurrence in the distance distribution data. (C) DEER data for the NpSRII-L159R1/NpHtrlI 157 complex reconstituted into SMALPs (blue) and nanodiscs (red). Experimental time domain data, $V(t)$, superimposed with the corresponding background fits (black). (D) Background-corrected and normalized form factors, $F(t)$, overlaid with the corresponding fits (black) calculated by Tikhonov regularization using DeerAnalysis. ${ }^{48}$. (E) Interspin distance distributions, normalized to unity, obtained by Tikhonov regularization using DeerAnalysis ${ }^{48}$ of the corresponding data shown in (D). (F) Normalized distance distributions obtained by MtsslWizard simulations for the computed model ${ }^{19}$ based on the " $\mathrm{V}$ "-shaped conformation (PDB: $\left.1 \mathrm{H} 2 \mathrm{~S}\right)^{1}$ (black), and for the " $\mathrm{U}$ "-shaped conformation (PDB: 5JJE) ${ }^{66}$ (green).

presented in Fig. 6E. The calculated intradimer distance distribution reveals three maxima at $\sim 2.6,3.7$ and $4.8 \mathrm{~nm}$ for the "V"-shaped conformation (Fig. 6E). According to the structural model shown in Fig. 6B, the minimum distance value of $\sim 2.6$ corresponds to the distance between spin label rotamers pointing to the center of the complex. The intermediate value of $\sim 3.7$ is a combination of the distances between the "inward" and the "outward" rotamers (or vice versa) of the two NpSRII molecules in the complex and the maximum value of $\sim 4.8$ marks the distance between rotamers oriented away from the center of the complex. For the "U"-shaped structure (PDB: 5JJE), the three respective maxima are found to be at $\sim 2.3,3.2$ and $4.2 \mathrm{~nm}$. Interestingly, all three corresponding distances values predicted for the "U"-shaped structure are shifted to lower values compared to the respective values for the "V"-shaped conformation revealing the more compact structure of the complex and facilitating clear discrimination between these two conformations.

The set of three distance peak values as predicted by in silico modeling for the "V"-shaped conformation agrees well with the 
experimental interspin distance distribution peaks obtained for both SMALPs and nanodiscs. However, the peak amplitudes resulting from the experiment and the simulation are different. The DEER experiment suggests the outward orientation of the spin label side chains to be dominantly populated, whereas in the model the probability for inward orientation of at least one of the spin labels is high leading to the large peak at $3.7 \mathrm{~nm}$.

All these data indicate that the $N p S R I I / N p H$ trII complex in SMALPs forms the two-dimer-of-dimers structure similar to that found in nanodiscs ${ }^{26}$ with the complex in the "V"-shaped conformation. Hence, the investigated SMALPs (see Fig. 6E) provide enough space for reconstitution of a single $2: 2 \mathrm{NpSRII} /$ NpHtrII dimer.

\section{Discussion}

A variety of processes in cell membranes is triggered by interactions of membrane proteins. To investigate such interactions in detail, the complexes of interacting proteins need to be isolated in their entirety and studied in a model membrane system. Detergents usually used for the solubilization and isolation of membrane proteins could negatively influence their stability and functionality. SMA copolymers have been shown to enable a direct detergent-free extraction of membrane proteins or their complexes together with patches of lipid bilayer yielding stable nanocontainers, in which lipid-encased membrane proteins could be studied. ${ }^{27,30-34,71}$

The archaeal photoreceptor/transducer complex, NpSRII/ $N p$ HtrII, provides a unique model system to study structural interactions between different integral membrane proteins and their conformational changes during the transmembrane signal transfer. ${ }^{11,72,73}$ Our study demonstrated the ability of the SMA polymer to extract the $\mathrm{NpSRII/NpHtrII}{ }_{157}$ complex from proteoliposomes in a detergent-free manner as has been shown for other membrane protein complexes. ${ }^{27,33,34}$ The EPR spectra and DEER measurements revealed that the structural integrity of the $N p S R I I / N p H_{t r I I}{ }_{157}$ complex reconstituted in SMALPs was preserved. SEC confirmed the stability of SMALP preparations. DLS measurements indicated the average particle size of assembled SMALPs to be $10 \mathrm{~nm}$, which corresponds to reported DLS values. ${ }^{30,53,55,74,75}$ TEM images showed the individual SMALPs as monodisperse particles with well-defined shape. The lateral size was in the range of $12-14 \mathrm{~nm}$, which is consistent with reported data of 10-15 nm obtained by TEM. ${ }^{27,30,32,76,77}$ The mean height, as measured by AFM is slightly less than the value of $4.6 \mathrm{~nm}$ obtained by small-angle neutron scattering experiments on SMALPs derived from DMPC vesicles. ${ }^{55}$ Such slight discrepancy can be attributed to the technical limitations of our AFM experiments.

Further, we applied spectroscopic methods for a more detailed structural characterization of SMALPs containing $N p$ SRII/NpHtrII ${ }_{157}$. RR spectroscopy enabled to characterize the

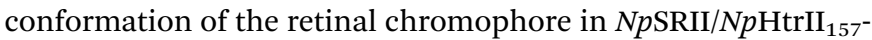
SMALPs due to the resonance enhancement of characteristic retinal chromophore bands that provide molecular fingerprinting. The RR spectroscopic data clearly indicated that alltrans retinal is the predominant isomer in reconstituted
NpSRII. Thus, reconstituted NpSRII retains its light-sensitive retinal prosthetic group with its functional conformation not affected upon the SMA-mediated extraction of the NpSRII/ $N p \mathrm{HtrII}_{157}$ complex.

We used cw EPR spectroscopy to analyse the protein dynamics through tracking the residual motion of spin labeled side chains. cw EPR spectra of NpSRII-L159R1/NpHtrII 157- $^{-}$ SMALP samples revealed that SMALPs generally maintained the dynamic features of the reconstituted $N p S R I I / N p H_{t r I I} I_{157}$ complex where the behaviour of the NpSRII-L159R1 side chain, either in complex with $\mathrm{NpHtrII} \mathrm{H}_{157}$ or without the transducer, was similar compared to that reconstituted in liposomes or nanodiscs. Hence, the conformation and dynamics of the NpSRII molecule in the vicinity of the functionally important helix F was not notably affected by the SMA polymer.

The DEER measurements showed that the interspin distance distribution in the SMALP-reconstituted NpSRII-L159R1/ $\mathrm{NpHtrII}$ complexes was similar to that measured with nanodiscs, which contained 2:2 NpSRII/NpHtrII dimers. ${ }^{26}$ The positions of the interspin distance peaks agree with results of $i n$ silico modeling of a single $2: 2 \mathrm{NpSRII/NpHtrII} \mathrm{dimer} \mathrm{for} \mathrm{the}$ "V"-shaped conformation. ${ }^{1}$ The difference in the peak amplitudes between simulation and experiment may indicate a more densely packed conformation of the $N p S R I I / N p$ HtrII-SMALP complex at the receptor-transducer interface, which renders inward orientations of the spin label side chain less probable. Hence, the DEER data indicate that $N p S R I I / N p H$ trII complexes incorporate into SMALPs as $2: 2$ dimers with the distances between L159R1 sites according to the "V"-shaped conformation. ${ }^{1}$

\section{Conclusions}

We have demonstrated that the $N p S R I I / N p H_{t r I I}{ }_{157}$ complex can be extracted from proteoliposomes in a detergent-free manner using the $3: 1$ SMA copolymer. The presented data indicate the integrity of this complex upon its reconstitution in SMA/lipid particles. Therefore, SMALPs that contain the NpSRII/NpHtrII complex can provide a valuable model system for studying the molecular events underlying the consecutive steps in receptortransducer signalling. Time resolved analyses of light induced conformational changes of this system are currently performed.

\section{Author contribution}

N. V., A. Y. M., K. V. S. and H. J. S. designed the study. N. V. expressed and purified proteins for assays, developed a protocol for the preparation of $N p S R I I / N p H_{t r I I}{ }_{157}$-nanodiscs, prepared samples of $N p S R I I / N p H_{t r I I}{ }_{157}$-SMALPs, contributed to the TEM, AFM and RR spectroscopy experiments, and drafted the manuscript. W. M. developed a protocol for the preparation of $N p$ SRII/N $p$ HtrII $_{157}$-SMALPs, prepared samples of NpSRII/ $N p \mathrm{HtrII}_{157}$-containing liposomes, performed the $\mathrm{cw}$ and pulsed EPR experiments and wrote the respective sections of the manuscript. A. C. performed structural modeling, carried out pulsed EPR experiments and wrote the respective sections of the manuscript. D. B., T. I., M. P. K. and K. V. S. performed the TEM 
and AFM experiments, and wrote the respective sections of the manuscript. A. A., V. B. and P. T. performed the resonance Raman spectroscopy experiments and wrote the respective sections of the manuscript. H. J. S. conceived, supervised and directed the project, analysed data, and wrote the final version of the manuscript. All authors read, edited and approved the final version of the manuscript.

\section{Conflicts of interest}

There are no conflicts to declare.

\section{Acknowledgements}

This work was supported by German Research Foundation (DFG, STE640/14), the Federal Ministry of Education and Research of Germany (BMBF, 05K14MPA), the German Academic Exchange Service (DAAD) and the grants from the Russian Science Foundation (14-50-00029 (K. S.)) and the Russian Foundation for Basic Research (RFBR) (Project No. 1554-12385 (K. S.), 16-02-00248 (P. T.)). We thank J. P. Klare for helpful discussions.

\section{Notes and references}

1 V. I. Gordeliy, J. Labahn, R. Moukhametzianov, R. Efremov, J. Granzin, R. Schlesinger, G. Buldt, T. Savopol, A. J. Scheidig, J. P. Klare and M. Engelhard, Nature, 2002, 419, 484-487.

2 A. Royant, P. Nollert, K. Edman, R. Neutze, E. M. Landau, E. Pebay-Peyroula and J. Navarro, Proc. Natl. Acad. Sci. U. S. A., 2001, 98, 10131-10136.

3 H. Luecke, B. Schobert, J. K. Lanyi, E. N. Spudich and J. L. Spudich, Science, 2001, 293, 1499-1503.

4 M. Grote, M. Engelhard and P. Hegemann, Biochim. Biophys. Acta, 2014, 1837, 533-545.

5 D. N. Shalaeva, M. Y. Galperin and A. Y. Mulkidjanian, Biol. Direct, 2015, 10, 63.

6 J. P. Klare, V. I. Gordeliy, J. Labahn, G. Buldt, H. J. Steinhoff and M. Engelhard, FEBS Lett., 2004, 564, 219-224.

7 A. A. Wegener, I. Chizhov, M. Engelhard and H. J. Steinhoff, J. Mol. Biol., 2000, 301, 881-891.

8 C. S. Yang and J. L. Spudich, Biochemistry, 2001, 40, 1420714214.

9 A. A. Wegener, J. P. Klare, M. Engelhard and H. J. Steinhoff, EMBO J., 2001, 20, 5312-5319.

10 K. Wuichet and I. B. Zhulin, Sci. Signaling, 2010, 3, ra50.

11 J. P. Klare, E. Bordignon, M. Engelhard and H. J. Steinhoff, Eur. J. Cell Biol., 2011, 90, 731-739.

12 J. P. Klare, E. Bordignon, M. Engelhard and H. J. Steinhoff, Photochem. Photobiol. Sci., 2004, 3, 543-547.

13 E. Bordignon, J. P. Klare, J. Holterhues, S. Martell, A. Krasnaberski, M. Engelhard and H. J. Steinhoff, Photochem. Photobiol., 2007, 83, 263-272.

14 R. Moukhametzianov, J. P. Klare, R. Efremov, C. Baeken, A. Goppner, J. Labahn, M. Engelhard, G. Buldt and V. I. Gordeliy, Nature, 2006, 440, 115-119.
15 A. Ishchenko, E. Round, V. Borshchevskiy, S. Grudinin, I. Gushchin, J. P. Klare, T. Balandin, A. Remeeva, M. Engelhard, G. Buldt and V. Gordeliy, J. Photochem. Photobiol., B, 2013, 123, 55-58.

16 J. J. Falke and G. L. Hazelbauer, Trends Biochem. Sci., 2001, 26, 257-265.

17 K. M. Ottemann, W. Z. Xiao, Y. K. Shin and D. E. Koshland, Science, 1999, 285, 1751-1754.

18 D. Q. Yu, X. M. Ma, Y. H. Tu and L. H. Lai, Sci. Rep., 2015, 5, 8640.

19 P. S. Orekhov, D. Klose, A. Y. Mulkidjanian, K. V. Shaitan, M. Engelhard, J. P. Klare and H. J. Steinhoff, PLoS Comput. Biol., 2015, 11, e1004561.

20 W. D. Hoff, K. H. Jung and J. L. Spudich, Annu. Rev. Biophys. Biomol. Struct., 1997, 26, 223-258.

21 J. L. Spudich, C. S. Yang, K. H. Jung and E. N. Spudich, Annu. Rev. Cell Dev. Biol., 2000, 16, 365-392.

22 J. P. Klare, I. Chizhov and M. Engelhard, Results Probl. Cell Differ., 2008, 45, 73-122.

23 I. P. Hohenfeld, A. A. Wegener and M. Engelhard, FEBS Lett., 1999, 442, 198-202.

24 P. Orekhov, A. Bothe, H. J. Steinhoff, K. V. Shaitan, S. Raunser, D. Fotiadis, R. Schlesinger, J. P. Klare and M. Engelhard, Photochem. Photobiol., 2017, 93, 796-804.

25 J. J. Falke and K. N. Piasta, Curr. Opin. Struct. Biol., 2014, 29, 85-94.

26 I. Orban-Glass, N. Voskoboynikova, K. B. Busch, D. Klose, C. Rickert, W. Mosslehy, F. Roder, V. Wilkens, J. Piehler, M. Engelhard, H. J. Steinhoff and J. P. Klare, Biochemistry, 2015, 54, 349-362.

27 A. R. Long, C. C. O'Brien, K. Malhotra, C. T. Schwall, A. D. Albert, A. Watts and N. N. Alder, BMC Biotechnol., 2013, 13, 41.

28 J. M. Dorr, S. Scheidelaar, M. C. Koorengevel, J. J. Dominguez, M. Schafer, C. A. van Walree and J. A. Killian, Eur. Biophys. J., 2016, 45, 3-21.

29 S. C. Lee and N. L. Pollock, Biochem. Soc. Trans., 2016, 44, 1011-1018.

30 T. J. Knowles, R. Finka, C. Smith, Y. P. Lin, T. Dafforn and M. Overduin, J. Am. Chem. Soc., 2009, 131, 7484-7485.

31 M. Orwick-Rydmark, J. E. Lovett, A. Graziadei, L. Lindholm, M. R. Hicks and A. Watts, Nano Lett., 2012, 12, 4687-4692.

32 D. J. Swainsbury, S. Scheidelaar, R. van Grondelle, J. A. Killian and M. R. Jones, Angew. Chem., Int. Ed. Engl., 2014, 53, 11803-11807.

33 J. M. Dorr, M. C. Koorengevel, M. Schafer, A. V. Prokofyev, S. Scheidelaar, E. A. van der Cruijsen, T. R. Dafforn, M. Baldus and J. A. Killian, Proc. Natl. Acad. Sci. U. S. A., 2014, 111, 18607-18612.

34 A. J. Bell, L. K. Frankel and T. M. Bricker, J. Biol. Chem., 2015, 290, 18429-18437.

35 R. C. Arenas, J. Klingler, C. Vargas and S. Keller, Nanoscale, 2016, 8, 15016-15026.

36 J. J. Dominguez Pardo, J. M. Dorr, A. Iyer, R. C. Cox, S. Scheidelaar, M. C. Koorengevel, V. Subramaniam and J. A. Killian, Eur. Biophys. J., 2017, 46, 91-101.

37 G. Jeschke, Annu. Rev. Phys. Chem., 2012, 63, 419-446. 
38 I. D. Sahu, R. M. McCarrick, K. R. Troxel, R. F. Zhang, H. J. Smith, M. M. Dunagan, M. S. Swartz, P. V. Rajan, B. M. Kroncke, C. R. Sanders and G. A. Lorigan, Biochemistry, 2013, 52, 6627-6632.

39 B. Ehresmann, P. Imbault and J. H. Weil, Anal. Biochem., 1973, 54, 454-463.

40 K. Shimono, M. Iwamoto, M. Sumi and N. Kamo, FEBS Lett., 1997, 420, 54-56.

41 N. Mennes, J. P. Klare, I. Chizhov, R. Seidel, R. Schlesinger and M. Engelhard, FEBS Lett., 2007, 581, 1487-1494.

42 T. H. Bayburt and S. G. Sligar, Proc. Natl. Acad. Sci. U. S. A., 2002, 99, 6725-6730.

43 C. A. Schneider, W. S. Rasband and K. W. Eliceiri, Nat. Methods, 2012, 9, 671-675.

44 N. V. Suetin, S. A. Evlashin, A. V. Egorov, K. V. Mironovich, S. A. Dagesyan, L. V. Yashina, E. A. Goodilin and V. A. Krivchenko, Phys. Chem. Chem. Phys., 2016, 18, 12344-12349.

45 D. Klose, N. Voskoboynikova, I. Orban-Glass, C. Rickert, M. Engelhard, J. P. Klare and H. J. Steinhoff, FEBS Lett., 2014, 588, 3970-3976.

46 R. E. Martin, M. Pannier, F. Diederich, V. Gramlich, M. Hubrich and H. W. Spiess, Angew. Chem., Int. Ed., 1998, 37, 2834-2837.

47 M. Pannier, S. Veit, A. Godt, G. Jeschke and H. W. Spiess, J. Magn. Reson., 2000, 142, 331-340.

48 G. Jeschke, V. Chechik, P. Ionita, A. Godt, H. Zimmermann, J. Banham, C. R. Timmel, D. Hilger and H. Jung, Appl. Magn. Reson., 2006, 30, 473-498.

49 G. Hagelueken, R. Ward, J. H. Naismith and O. Schiemann, Appl. Magn. Reson., 2012, 42, 377-391.

50 S. J. Hirst, N. Alexander, H. S. McHaourab and J. Meiler, J. Struct. Biol., 2011, 173, 506-514.

51 E. J. Hustedt, R. A. Stein, L. Sethaphong, S. Brandon, Z. Zhou and S. C. Desensi, Biophys. J., 2006, 90, 340-356.

52 Z. Zhou, S. C. DeSensi, R. A. Stein, S. Brandon, M. Dixit, E. J. McArdle, E. M. Warren, H. K. Kroh, L. Song, C. E. Cobb, E. J. Hustedt and A. H. Beth, Biochemistry, 2005, 44, 15115-15128.

53 S. Scheidelaar, M. C. Koorengevel, J. D. Pardo, J. D. Meeldijk, E. Breukink and J. A. Killian, Biophys. J., 2015, 108, 279-290.

54 D. V. Bagrov, N. Voskoboynikova, G. A. Armeev, W. Mosslehy,

G. S. Gluhov, T. T. Ismagulova, A. Y. Mulkidjanian, M. P. Kirpichnikov, H.-J. Steinhoff and K. V. Shaitan, Biofizika, 2016, 61, 942-949.

55 M. Jamshad, V. Grimard, I. Idini, T. J. Knowles, M. R. Dowle, N. Schofield, P. Sridhar, Y. P. Lin, R. Finka, M. Wheatley, O. R. T. Thomas, R. E. Palmer, M. Overduin, C. Govaerts, J. M. Ruysschaert, K. J. Edler and T. R. Dafforn, Nano Res., 2015, 8, 774-789.

56 T. H. Bayburt and S. G. Sligar, Protein Sci., 2003, 12, 24762481.

57 T. H. Bayburt, J. W. Carlson and S. G. Sligar, Langmuir, 2000, 16, 5993-5997.
58 C. D. Blanchette, J. A. Cappuccio, E. A. Kuhn, B. W. Segelke, W. H. Benner, B. A. Chromy, M. A. Coleman, G. Bench, P. D. Hoeprich and T. A. Sulchek, Biochim. Biophys. Acta, Biomembr., 2009, 1788, 724-731.

59 P. Samyn and G. Schoukens, Polymers, 2014, 6, 820-845.

60 B. Aton, A. G. Doukas, R. H. Callender, B. Becher and

T. G. Ebrey, Biochemistry, 1977, 16, 2995-2999.

61 C. Gellini, B. Luttenberg, J. Sydor, M. Engelhard and

P. Hildebrandt, FEBS Lett., 2000, 472, 263-266.

62 O. S. Mironova, R. G. Efremov, B. Person, J. Heberle, I. L. Budyak, G. Buldt and R. Schlesinger, FEBS Lett., 2005, 579, 3147-3151.

63 T. Alshuth, M. Stockburger, P. Hegemann and D. Oesterhelt, FEBS Lett., 1985, 179, 55-59.

64 S. O. Smith, A. B. Myers, R. A. Mathies, J. A. Pardoen, C. Winkel, E. M. van den Berg and J. Lugtenburg, Biophys. J., 1985, 47, 653-664.

65 S. O. Smith, J. A. Pardoen, J. Lugtenburg and R. A. Mathies, J. Phys. Chem., 1987, 91, 804-819.

66 E. Bordignon and Y. Polyhach, Methods Mol. Biol., 2013, 974, 329-355.

67 H. J. Steinhoff, Biol. Chem., 2004, 385, 913-920.

68 G. W. Reginsson and O. Schiemann, Biochem. J., 2011, 434, 353-363.

69 A. Ishchenko, E. Round, V. Borshchevskiy, S. Grudinin, I. Gushchin, J. P. Klare, A. Remeeva, V. Polovinkin, P. Utrobin, T. Balandin, M. Engelhard, G. Buldt and V. Gordeliy, Sci. Rep., 2017, 7, 41811.

70 A. Ishchenko, E. Round, V. Borshchevskiy, S. Grudinin, I. Gushchin, J. P. Klare, A. Remeeva, V. Polovinkin, P. Utrobin, T. Balandin, M. Engelhard, G. Büldt and V. Gordeliy, Sci. Rep., 2017, 7, 41811.

71 C. Logez, M. Damian, C. Legros, C. Dupre, M. Guery, S. Mary, R. Wagner, C. M'Kadmi, O. Nosjean, B. Fould, J. Marie, J. A. Fehrentz, J. Martinez, G. Ferry, J. A. Boutin and J. L. Baneres, Biochemistry, 2016, 55, 38-48.

72 J. P. Klare, E. Bordignon, M. Doebber, J. Fitter, J. Kriegsmann, I. Chizhov, H. J. Steinhoff and M. Engelhard, J. Mol. Biol., 2006, 356, 1207-1221.

73 J. Holterhues, E. Bordignon, D. Klose, C. Rickert, J. P. Klare, S. Martell, L. Li, M. Engelhard and H. J. Steinhoff, Biophys. J., 2011, 100, 2275-2282.

74 M. C. Orwick, P. J. Judge, J. Procek, L. Lindholm, A. Graziadei, A. Engel, G. Grobner and A. Watts, Angew. Chem., Int. Ed. Engl., 2012, 51, 4653-4657.

75 R. Zhang, I. D. Sahu, L. Liu, A. Osatuke, R. G. Comer, C. Dabney-Smith and G. A. Lorigan, Biochim. Biophys. Acta, 2015, 1848, 329-333.

76 V. Postis, S. Rawson, J. K. Mitchell, S. C. Lee, R. A. Parslow, T. R. Dafforn, S. A. Baldwin and S. P. Muench, Biochim. Biophys. Acta, 2015, 1848, 496-501.

77 S. Gulati, M. Jamshad, T. J. Knowles, K. A. Morrison, R. Downing, N. Cant, R. Collins, J. B. Koenderink, R. C. Ford, M. Overduin, I. D. Kerr, T. R. Dafforn and A. J. Rothnie, Biochem. J., 2014, 461, 269-278. 